\title{
Some Properties for Strong Differential Subordination of Analytic Functions Associated with Wanas Operator
}

\author{
Abbas Kareem Wanas ${ }^{1}$ and Pall-Szabo Agnes Orsolya ${ }^{2}$ \\ ${ }^{1}$ Department of Mathematics, College of Science, University of Al-Qadisiyah, Iraq \\ e-mail: abbas.kareem.w@qu.edu.iq \\ ${ }^{2}$ Department of Mathematics, Faculty of Mathematics and Computer Science, \\ Babes-Bolyai University, Cluj-Napoca, Romania \\ e-mail: pallszaboagnes@math.ubbcluj.ro
}

\begin{abstract}
In this paper, by making use of Wanas operator, we derive some properties related to the strong differential subordinations of analytic functions defined in the open unit disk and closed unit disk of the complex plane.
\end{abstract}

\section{Introduction}

Indicate by $\mathcal{H}(U \times \bar{U})$ the family of all analytic functions in $U \times \bar{U}$. Let $U=\{z \in \mathbb{C}:|z|<1\}$ and $\bar{U}=\{z \in \mathbb{C}:|z| \leq 1\}$ denote the open unit disk and the closed unit disk of the complex plane, respectively. For $n \in \mathbb{N}=\{1,2, \ldots\}$ and $a \in \mathbb{C}$, let

$$
\begin{gathered}
\mathcal{H}^{*}[a, n, \zeta]=\left\{f \in \mathcal{H}(U \times \bar{U}): f(z, \zeta)=a+a_{n}(\zeta) z^{n}+a_{n+1}(\zeta) z^{n+1}+\cdots,\right. \\
z \in U, \zeta \in \bar{U}\}
\end{gathered}
$$

where $a_{k}(\zeta)$ are holomorphic functions in $\bar{U}$ for $k \geq n$.

Received: December 30, 2019; Accepted: March 28, 2020

2010 Mathematics Subject Classification: 30C45.

Keywords and phrases: analytic function, convex function, strong differential subordination, Wanas operator. Copyright (C) 2020 Abbas Kareem Wanas and Pall-Szabo Agnes Orsolya. This is an open access article distributed under the Creative Commons Attribution License, which permits unrestricted use, distribution, and reproduction in any medium, provided the original work is properly cited. 
Also, let $\mathcal{A}_{n \zeta}^{*}=\left\{f \in \mathcal{H}(U \times \bar{U}): f(z, \zeta)=z+a_{n+1}(\zeta) z^{n+1}+\cdots, z \in U, \zeta \in \bar{U}\right\}$, where $a_{k}(\zeta)$ are holomorphic functions in $\bar{U}$ for $k \geq n+1$.

A function $f \in \mathcal{H}^{*}[a, n, \zeta]$ is said to be starlike in $U \times \bar{U}$ if

$$
\operatorname{Re}\left\{\frac{z f_{z}^{\prime}(z, \zeta)}{f(z, \zeta)}\right\}>0, \quad(z \in U, \zeta \in \bar{U})
$$

Denote the class of all starlike functions in $U \times \bar{U}$ by $S_{\zeta}^{*}$.

Similar, $f \in \mathcal{H}^{*}[a, n, \zeta]$ is said to be convex in $U \times \bar{U}$ if

$$
\operatorname{Re}\left\{\frac{z f_{z}^{\prime \prime 2}(z, \zeta)}{f_{z}^{\prime}(z, \zeta)}+1\right\}>0, \quad(z \in U, \zeta \in \bar{U})
$$

Denote the class of all convex functions in $U \times \bar{U}$ by $K_{\zeta}^{*}$.

Definition 1.1 [9]. Let $f(z, \zeta), g(z, \zeta)$ be analytic in $U \times \bar{U}$. The function $f(z, \zeta)$ is said to be strongly subordinate to $g(z, \zeta)$, written $f(z, \zeta) \prec \prec F(z, \zeta), \quad z \in U$, $\zeta \in \bar{U}$, if there exists an analytic function $w$ in $U$ with $w(0)=0$ and $|w(z)|<1, z \in U$ such that $f(z, \zeta)=g(w(z), \zeta)$ for all $\zeta \in \bar{U}$.

\section{Remark 1.1 [9].}

(1) Since $f(z, \zeta)$ is analytic in $U \times \bar{U}$, for all $\zeta \in \bar{U}$ and univalent in $U$, for all $\zeta \in \bar{U}$, Definition 1.1 is equivalent to $f(0, \zeta)=g(0, \zeta)$ for all $\zeta \in \bar{U}$ and $f(U \times \bar{U}) \subset g(U \times \bar{U})$.

(2) If $f(z, \zeta)=f(z)$ and $g(z, \zeta)=g(z)$, then the strong subordination becomes the usual notion of subordination.

Let $\mathcal{A}_{\zeta}^{*}$ denote the subclass of the functions $f(z, \zeta) \in \mathcal{H}(U \times \bar{U})$ of the form:

$$
f(z, \zeta)=z+\sum_{k=2}^{\infty} a_{k}(\zeta) z^{k}, \quad z \in U, \zeta \in \bar{U}
$$

which are analytic and univalent in $U \times \bar{U}$. 
For $\alpha \in \mathbb{R}, \beta \geq 0$ with $\alpha+\beta>0, l \in \mathbb{N}, m, \lambda \in \mathbb{N}_{0}=\mathbb{N} \cup\{0\}$ and $f \in \mathcal{A}$, the Wanas operator $W_{\alpha, \beta}^{l, \lambda}: \mathcal{A}_{\zeta}^{*} \rightarrow \mathcal{A}_{\zeta}^{*}$, (see [12]) is defined by

$$
W_{\alpha, \beta}^{l, \lambda} f(z, \zeta)=z+\sum_{k=2}^{\infty}\left[\sum_{m=1}^{l}\left(\begin{array}{l}
l \\
m
\end{array}\right)(-1)^{m+1}\left(\frac{\alpha^{m}+n \beta^{m}}{\alpha^{m}+\beta^{m}}\right)\right]^{\lambda} a_{k}(\zeta) z^{n} .
$$

It is easily verified from (1.2) that

$$
\begin{aligned}
z\left(W_{\alpha, \beta}^{l, \lambda} f(z, \zeta)\right)^{\prime}= & {\left[\sum_{m=1}^{l}\left(\begin{array}{l}
l \\
m
\end{array}\right)(-1)^{m+1}\left(\left(\frac{\alpha}{\beta}\right)^{m}+1\right) W_{\alpha, \beta}^{l, \lambda+1} f(z, \zeta)\right.} \\
& -\left[\sum_{m=1}^{l}\left(\begin{array}{l}
l \\
m
\end{array}\right)(-1)^{m+1}\left(\frac{\alpha}{\beta}\right)^{m}\right] W_{\alpha, \beta}^{l, \lambda} f(z, \zeta) .
\end{aligned}
$$

Some of the special cases of the operator defined by (1.2) can be found in $[1,2,4,10$, $11]$.

In recent years, many authors obtained various interesting results associated with strong differential subordination and superordination for example (see $[3,5,6,13,14]$ ).

In order to derive our main results, we need the following lemmas.

Lemma 1.1 [8]. Let $h(z, \zeta)$ be a convex function with $h(0, \zeta)=a$, for every $\zeta \in \bar{U}$ and let $\gamma \in \mathbb{C}^{*}=\mathbb{C} \backslash\{0\}$ with $\operatorname{Re}(\gamma) \geq 0$. If $p \in \mathcal{H}^{*}[a, n, \zeta]$ and

$$
p(z, \zeta)+\frac{1}{\gamma} z p_{z}^{\prime}(z, \zeta) \prec \prec h(z, \zeta), \quad(z \in U, \zeta \in \bar{U}),
$$

then

$$
p(z, \zeta) \prec \prec q(z, \zeta) \prec \prec h(z, \zeta), \quad(z \in U, \zeta \in \bar{U}),
$$

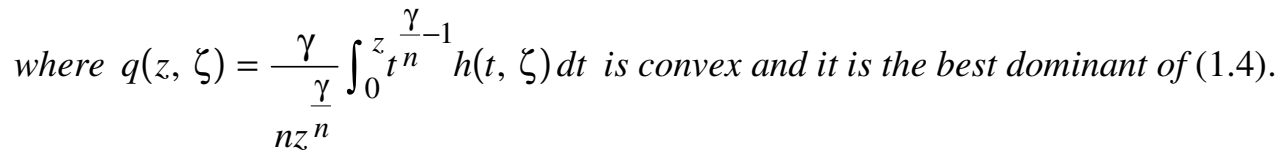

Lemma 1.2 [7]. Let $q(z, \zeta)$ be a convex function in $U \times \bar{U}$ for all $\zeta \in \bar{U}$ and let $h(z, \zeta)=q(z, \zeta)+n \delta z q_{z}^{\prime}(z, \zeta), z \in U, \zeta \in \bar{U}$, where $\delta>0$ and $n$ is a positive integer. 
If

$$
p(z, \zeta)=q(0, \zeta)+p_{n}(\zeta) z^{n}+p_{n+1}(\zeta) z^{n+1}+\cdots,
$$

is analytic in $U \times \bar{U}$ and

$$
p(z, \zeta)+\delta z p_{z}^{\prime}(z, \zeta) \prec \prec h(z, \zeta), \quad(z \in U, \zeta \in \bar{U}),
$$

then

$$
p(z, \zeta) \prec \prec q(z, \zeta), \quad(z \in U, \zeta \in \bar{U})
$$

and this result is sharp.

\section{Main Results}

Theorem 2.1. Let $h(z, \zeta)$ be a convex function such that $h(0, \zeta)=1$. If $f \in \mathcal{A}_{\zeta}^{*}$ satisfies the strong differential subordination:

$$
\left(W_{\alpha, \beta}^{l, \lambda} f(z, \zeta)\right)_{z}^{\prime} \prec \prec h(z, \zeta)
$$

then

$$
\frac{W_{\alpha, \beta}^{l, \lambda} f(z, \zeta)}{z} \prec \prec q(z, \zeta) \prec \prec h(z, \zeta),
$$

where $q(z, \zeta)=\frac{1}{z} \int_{0}^{z} h(t, \zeta) d t$ is convex and it is the best dominant.

Proof. Suppose that

$$
p(z, \zeta)=\frac{W_{\alpha, \beta}^{l, \lambda} f(z, \zeta)}{z}, \quad z \in U, \zeta \in \bar{U}
$$

Then the function $p(z, \zeta)$ is analytic in $U \times \bar{U}$ and $p(0, \zeta)=1$.

Simple computations from (2.2), we get

$$
p(z, \zeta)+z p_{z}^{\prime}(z, \zeta)=\left(W_{\alpha, \beta}^{l, \lambda} f(z, \zeta)\right)_{z}^{\prime} .
$$


Using (2.3), (2.1) becomes

$$
p(z, \zeta)+z p_{z}^{\prime}(z, \zeta) \prec \prec h(z, \zeta) .
$$

An application of Lemma 1.1 with $n=1, \gamma=1$ yields

$$
\frac{W_{\alpha, \beta}^{l, \lambda} f(z, \zeta)}{z} \prec \prec q(z, \zeta)=\frac{1}{z} \int_{0}^{z} h(t, \zeta) d t \prec \prec h(z, \zeta)
$$

By taking $h(z, \zeta)=\frac{\zeta+(2 \rho-\zeta) z}{1+z}, 0 \leq \rho<1$ in Theorem 2.1, we obtain the following corollary:

Corollary 2.1. If $f \in \mathcal{A}_{\zeta}^{*}$ satisfies the strong differential subordination:

$$
\left(W_{\alpha, \beta}^{l, \lambda} f(z, \zeta)\right)_{z}^{\prime} \prec \prec \frac{\zeta+(2 \rho-\zeta) z}{1+z},
$$

then

$$
\frac{W_{\alpha, \beta}^{l, \lambda} f(z, \zeta)}{z} \prec \prec \frac{1}{z} \int_{0}^{z} \frac{\zeta+(2 \rho-\zeta) t}{1+t} d t=2 \rho-\zeta+\frac{2(\zeta-\rho)}{z} \ln (1+z)
$$

Theorem 2.2. Let $h(z, \zeta)$ be a convex function such that $h(0, \zeta)=1$. If $0 \leq \sigma<p$, $\theta \in \mathbb{C}$ and $f \in \mathcal{A}_{\zeta}^{*}$ satisfies the strong differential subordination:

$$
\frac{1-\theta}{1-\sigma}\left(\frac{W_{\alpha, \beta}^{l, \lambda} f(z, \zeta)}{z}-\sigma\right)+\frac{\theta}{1-\sigma}\left(\left(W_{\alpha, \beta}^{l, \lambda} f(z, \zeta)\right)_{z}^{\prime}-\sigma\right) \prec \prec h(z, \zeta)
$$

then

$$
\frac{1}{1-\sigma}\left(\frac{W_{\alpha, \beta}^{l, \lambda} f(z, \zeta)}{z}-\sigma\right) \prec \prec q(z, \zeta) \prec \prec h(z, \zeta),
$$

where $q(z, \zeta)=\frac{1}{\theta} z^{-\frac{1}{\theta}} \int_{0}^{z} t^{\frac{1}{\theta}-1} h(t, \zeta) d t$ is convex and it is the best dominant. 
Proof. Suppose that

$$
p(z, \zeta)=\frac{1}{1-\sigma}\left(\frac{W_{\alpha, \beta}^{l, \lambda} f(z, \zeta)}{z}-\sigma\right), z \in U, \zeta \in \bar{U}
$$

Then the function $p(z, \zeta)$ is analytic in $U \times \bar{U}$ and $p(0, \zeta)=1$.

Differentiating both sides of (2.5) with respect to $z$, we have

$$
p(z, \zeta)+\theta z p_{z}^{\prime}(z, \zeta)=\frac{1-\theta}{1-\sigma}\left(\frac{W_{\alpha, \beta}^{l, \lambda} f(z, \zeta)}{z}-\sigma\right)+\frac{\theta}{1-\sigma}\left(\left(W_{\alpha, \beta}^{l, \lambda} f(z, \zeta)\right)_{z}^{\prime}-\sigma\right) .
$$

From (2.4) and (2.6), we get

$$
p(z, \zeta)+\theta z p_{z}^{\prime}(z, \zeta) \prec \prec h(z, \zeta) .
$$

An application of Lemma 1.1 with $n=1, \gamma=\frac{1}{\theta}$ yields

$$
\frac{1}{1-\sigma}\left(\frac{W_{\alpha, \beta}^{l, \lambda} f(z, \zeta)}{z}-\sigma\right) \prec \prec q(z, \zeta)=\frac{1}{\theta} z^{-\frac{1}{\theta}} \int_{0}^{z} t^{\frac{1}{\theta}-1} h(t, \zeta) d t \prec \prec h(z, \zeta) .
$$

Theorem 2.3. Let $q(z, \zeta)$ be a convex function such that $q(0, \zeta)=1$ and let $h$ be the function $h(z, \zeta)=q(z, \zeta)+z q_{z}^{\prime}(z, \zeta)$. If $f \in \mathcal{A}_{\zeta}^{*}$ satisfies the strong differential subordination:

$$
\left(\frac{z W_{\alpha, \beta}^{l, \lambda+1} f(z, \zeta)}{W_{\alpha, \beta}^{l, \lambda} f(z, \zeta)}\right)_{z}^{\prime} \prec \prec h(z, \zeta)
$$

then

$$
\frac{W_{\alpha, \beta}^{l, \lambda+1} f(z, \zeta)}{W_{\alpha, \beta}^{l, \lambda} f(z, \zeta)} \prec \prec q(z, \zeta)
$$

Proof. Suppose that 


$$
p(z, \zeta)=\frac{W_{\alpha, \beta}^{l, \lambda+1} f(z, \zeta)}{W_{\alpha, \beta}^{l, \lambda} f(z, \zeta)}, z \in U, \zeta \in \bar{U}
$$

Then the function $p(z, \zeta)$ is analytic in $U \times \bar{U}$ and $p(0, \zeta)=1$.

Differentiating both sides of (2.8) with respect to $z$ and using (2.7), we have

$$
\begin{aligned}
& p(z, \zeta)+z p_{z}^{\prime}(z, \zeta) \\
= & \frac{W_{\alpha, \beta}^{l, \lambda+1} f(z, \zeta)}{W_{\alpha, \beta}^{l, \lambda} f(z, \zeta)} \\
& +\frac{W_{\alpha, \beta}^{l, \lambda} f(z, \zeta)\left(W_{\alpha, \beta}^{l, \lambda+1} f(z, \zeta)\right)_{z}^{\prime}-W_{\alpha, \beta}^{l, \lambda+1} f(z, \zeta)\left(W_{\alpha, \beta}^{l, \lambda} f(z, \zeta)\right)_{z}^{\prime}}{\left[W_{\alpha, \beta}^{l, \lambda} f(z, \zeta)\right]^{2}} \\
= & \frac{W_{\alpha, \beta}^{l, \lambda} f(z, \zeta)\left(z W_{\alpha, \beta}^{l, \lambda+1} f(z, \zeta)\right)_{z}^{\prime}-z W_{\alpha, \beta}^{l, \lambda+1} f(z, \zeta)\left(W_{\alpha, \beta}^{l, \lambda} f(z, \zeta)\right)_{z}^{\prime}}{\left[W_{\alpha, \beta}^{l, \lambda} f(z, \zeta)\right]^{2}} \\
= & \left(\frac{z W_{\alpha, \beta}^{l, \lambda+1} f(z, \zeta)}{W_{\alpha, \beta}^{l, \lambda} f(z, \zeta)}\right)_{z} \prec \prec h(z, \zeta) .
\end{aligned}
$$

An application of Lemma 1.2, we obtain

$$
\frac{W_{\alpha, \beta}^{l, \lambda+1} f(z, \zeta)}{W_{\alpha, \beta}^{l, \lambda} f(z, \zeta)} \prec \prec q(z, \zeta) .
$$

Theorem 2.4. Let $q(z, \zeta)$ be a convex function such that $q(0, \zeta)=1$ and let $h$ be the function

$$
h(z, \zeta)=q(z, \zeta)+\frac{1}{1+\sum_{m=1}^{l}\left(\begin{array}{l}
l \\
m
\end{array}\right)(-1)^{m+1}\left(\left(\frac{\alpha}{\beta}\right)^{m}+1\right)} z q_{z}^{\prime}(z, \zeta)
$$

where $\sum_{m=1}^{l}\left(\begin{array}{c}l \\ m\end{array}\right)(-1)^{m+1}\left(\left(\frac{\alpha}{\beta}\right)^{m}+1\right)>0$. Suppose that 


$$
\begin{aligned}
F(z, \zeta)= & \frac{1+\sum_{m=1}^{l}\left(\begin{array}{l}
l \\
m
\end{array}\right)(-1)^{m+1}\left(\left(\frac{\alpha}{\beta}\right)^{m}+1\right)}{z_{m=1}^{l}\left(\begin{array}{l}
l \\
m
\end{array}\right)(-1)^{m+1}\left(\left(\frac{\alpha}{\beta}\right)^{m}+1\right)} \\
& \times \int_{0}^{z} t^{\sum_{m=1}^{l}\left(\begin{array}{l}
l \\
m
\end{array}\right)(-1)^{m+1}\left(\left(\frac{\alpha}{\beta}\right)^{m}+1\right)} f(t, \zeta) d t
\end{aligned}
$$

If $f \in \mathcal{A}_{\zeta}^{*}$ satisfies the strong differential subordination

$$
\left(W_{\alpha, \beta}^{l, \lambda} f(z, \zeta)\right)_{z}^{\prime} \prec \prec h(z, \zeta),
$$

then

$$
\left(W_{\alpha, \beta}^{l, \lambda} F(z, \zeta)\right)_{z}^{\prime} \prec \prec q(z, \zeta)
$$

Proof. Suppose that

$$
p(z, \zeta)=\left(W_{\alpha, \beta}^{l, \lambda} F(z, \zeta)\right)_{z}^{\prime}, \quad z \in U, \zeta \in \bar{U} .
$$

Then the function $p(z, \zeta)$ is analytic in $U \times \bar{U}$ and $p(0, \zeta)=1$.

From (2.9), we have

$$
\begin{aligned}
z^{\sum_{m=1}^{l}\left(\begin{array}{l}
l \\
m
\end{array}\right)(-1)^{m+1}\left(\left(\frac{\alpha}{\beta}\right)^{m}+1\right)} F(z, \zeta) \\
=\left(1+\sum_{m=1}^{l}\left(\begin{array}{c}
l \\
m
\end{array}\right)(-1)^{m+1}\left(\left(\frac{\alpha}{\beta}\right)^{m}+1\right)\right) \int_{0}^{z} t^{\sum_{m=1}^{l}\left(\begin{array}{c}
l \\
m
\end{array}\right)(-1)^{m+1}\left(\left(\frac{\alpha}{\beta}\right)^{m}+1\right)-1} f(t, \zeta) d t .
\end{aligned}
$$

Differentiating both sides of (2.12) with respect to $z$, we get

$$
\begin{aligned}
& \left(1+\sum_{m=1}^{l}\left(\begin{array}{c}
l \\
m
\end{array}\right)(-1)^{m+1}\left(\left(\frac{\alpha}{\beta}\right)^{m}+1\right)\right) f(z, \zeta) \\
= & \sum_{m=1}^{l}\left(\begin{array}{l}
l \\
m
\end{array}\right)(-1)^{m+1}\left(\left(\frac{\alpha}{\beta}\right)^{m}+1\right) F(z, \zeta)+z F_{z}^{\prime}(z, \zeta)
\end{aligned}
$$


and

$$
\begin{aligned}
& \left(1+\sum_{m=1}^{l}\left(\begin{array}{c}
l \\
m
\end{array}\right)(-1)^{m+1}\left(\left(\frac{\alpha}{\beta}\right)^{m}+1\right)\right) W_{\alpha, \beta}^{l, \lambda} f(z, \zeta) \\
= & \sum_{m=1}^{l}\left(\begin{array}{c}
l \\
m
\end{array}\right)(-1)^{m+1}\left(\left(\frac{\alpha}{\beta}\right)^{m}+1\right) W_{\alpha, \beta}^{l, \lambda} F(z, \zeta)+z\left(W_{\alpha, \beta}^{l, \lambda} F(z, \zeta)\right)_{z}^{\prime} .
\end{aligned}
$$

So

$$
\left(W_{\alpha, \beta}^{l, \lambda} f(z, \zeta)\right)_{z}^{\prime}=\left(W_{\alpha, \beta}^{l, \lambda} F(z, \zeta)\right)_{z}^{\prime}+\frac{z\left(W_{\alpha, \beta}^{l, \lambda} F(z, \zeta)\right)_{z^{2}}^{\prime \prime}}{1+\sum_{m=1}^{l}\left(\begin{array}{c}
l \\
m
\end{array}\right)(-1)^{m+1}\left(\left(\frac{\alpha}{\beta}\right)^{m}+1\right)}
$$

From (2.11) and (2.13), we obtain

$$
p(z, \zeta)=\frac{1}{1+\sum_{m=1}^{l}\left(\begin{array}{l}
l \\
m
\end{array}\right)(-1)^{m+1}\left(\left(\frac{\alpha}{\beta}\right)^{m}+1\right)} z p_{z}^{\prime}(z, \zeta)=\left(W_{\alpha, \beta}^{l, \lambda} f(z, \zeta)\right)_{z}^{\prime} .
$$

Using (2.14), (2.10) becomes

$$
\begin{aligned}
p(z, \zeta) & =\frac{1}{1+\sum_{m=1}^{l}\left(\begin{array}{l}
l \\
m
\end{array}\right)(-1)^{m+1}\left(\left(\frac{\alpha}{\beta}\right)^{m}+1\right)} z p_{z}^{\prime}(z, \zeta) \\
& \prec q(z, \zeta)+\frac{1}{1+\sum_{m=1}^{l}\left(\begin{array}{l}
l \\
m
\end{array}\right)(-1)^{m+1}\left(\left(\frac{\alpha}{\beta}\right)^{m}+1\right)} z q_{z}^{\prime}(z, \zeta) .
\end{aligned}
$$

An application of Lemma 1.2 yields $p(z, \zeta) \prec \prec q(z, \zeta)$. By using (2.10), we obtain

$$
\left(W_{\alpha, \beta}^{l, \lambda} F(z, \zeta)\right)_{z}^{\prime} \prec \prec q(z, \zeta) .
$$

\section{References}

[1] F. M. Al-Oboudi, On univalent functions defined by a generalized Salagean operator, Int. J. Math. Math. Sci. 27 (2004), 1429-1436. https://doi.org/10.1155/S0161171204108090 
[2] N. E. Cho and T. H. Kim, Multiplier transformations and strongly close-to-convex functions, Bull. Korean Math. Soc. 40(3) (2003), 399-410.| https://doi.org/10.4134/BKMS.2003.40.3.399

[3] N. E. Cho, O. S. Kwon and H. M. Srivastava, Strong differential subordination and superordination for multivalently meromorphic functions involving the Liu-Srivastava operator, Integral Transforms Spec. Funct. 21(8) (2010), 589-601. https://doi.org/10.1080/10652460903494751

[4] N. E. Cho and H. M. Srivastava, Argument estimates of certain analytic functions defined by a class of multiplier transformations, Math. Comput. Modelling 37(1-2) (2003), 39-49. https://doi.org/10.1016/S0895-7177(03)80004-3

[5] M. P. Jeyaraman and T. K. Suresh, Strong differential subordination and superordination of analytic functions, J. Math. Anal. Appl. 385(2) (2012), 854-864. https://doi.org/10.1016/j.jmaa.2011.07.016

[6] A. A. Lupas, A note on strong differential superordinations using a generalized Salagean operator and Ruscheweyh operator, Stud. Univ. Babes-Bolyai Math. 57(2) (2012), 135165.

[7] S. S. Miller and P. T. Mocanu, On some classes of first-order differential subordinations, Michigan Math. J. 32(2) (1985), 185-195. https://doi.org/10.1307/mmj/1029003185

[8] S. S. Miller and P. T. Mocanu, Differential subordinations: theory and applications, Monographs and Textbooks in Pure and Applied Mathematics, Vol. 225, Marcel Dekker Inc., New York and Basel, 2000. https://doi.org/10.1201/9781482289817

[9] G. I. Oros and Gh. Oros, Strong differential subordination, Turk. J. Math. 33 (2009), 249257.

[10] G. S. Salagean, Subclasses of univalent functions, Lecture Notes in Math., Vol. 1013, Springer Verlag, Berlin, 1983, pp. 362-372. https://doi.org/10.1007/BFb0066543

[11] S. R. Swamy, Inclusion properties of certain subclasses of analytic functions, Int. Math. Forum 7(36) (2012), 1751-1760.

[12] A. K. Wanas, New differential operator for holomorphic functions, Earthline J. Math. Sci. 2(2) (2019), 527-537. https://doi.org/10.34198/ejms.2219.527537

[13] A. K. Wanas and B. A. Frasin, Strong differential sandwich results for Frasin operator, Earthline J. Math. Sci. 3(1) (2020), 95-104. https://doi.org/10.34198/ejms.3120.95104

[14] A. K. Wanas and A. A. Lupas, On a new strong differential subordinations and superordinations of analytic functions involving the generalized differential operator, Int. J. Pure Appl. Math. 116(3) (2017), 571-579. 